\title{
Algo viejo, algo nuevo y ¿algo lento?
}

\author{
Marta Sánchez García ${ }^{\mathrm{a}}$, Ileana Gabriela Tomoiuª, Federico Hoyo Gómez ${ }^{\mathrm{b}}$ \\ y José Andrés Delgado Casado ${ }^{c}$
}

a M.I.R. de Medicina Familiar y Comunitaria. Centro de Salud Ciudad Real II. Ciudad Real (España).

${ }^{\mathrm{b}}$ Médico de Familia. Centro de Salud de Calzada de Calatrava. Ciudad Real (España).

c Médico de Familia. Centro de Salud Ciudad Real II.

Ciudad Real (España).

\section{Correspondencia:}

Marta Sánchez García.

Centro de Salud Ciudad Real II.

C/ Palma, 11. C.P. 13001.

Ciudad Real (España).

Correo electrónico:

fenetra_444@hotmail.com.

Este trabajo ha sido presentado como Comunicación Oral en el V Congreso SEMERGEN Castilla-La Mancha, celebrado en Talavera de la Reina

(Toledo - España), los días 7 y 8 de marzo de 2014.

Recibido el 22 de marzo de 2014.

Aceptado para su publicación el 27 de abril de 2014 .

\section{RESUMEN}

La fibrilación auricular (FA) es la arritmia más frecuente en la práctica clínica. Es responsable de una elevada morbilidad y mortalidad. Un paciente con FA conocida, que sigue tratamiento adecuadamente, y comienza con mareo, debe alertar al Médico de Familia. Un electrocardiograma es fundamental para un correcto diagnóstico: la presencia de una bradicardia en un paciente con FA. Esta situación clínica constituye una causa de derivación hospitalaria no demorable para realizar estudio etiológico y tratamiento adecuado ante la gravedad del cuadro clínico.

Palabras Clave. Fibrilación Auricular. Bradicardia. Bloqueo Auriculoventricular.

\section{ABSTRACT}

Something old, something new and... something slow?

Atrial fibrillation (AT) is the most frequent type of arrhythmia in clinical practice. It causes a high rate of morbidity and mortality. A patient with known AF who follows adequate therapy and starts feeling dizzy, should alert his/her GP. An electrocardiogram is essential for correct diagnosis: the presence of bradycardia in a patient with AF. This clinical situation constitutes a non-delayable reason to refer the patient to the hospital in order to carry out an etiologic study and adequate therapy because of the seriousness of this clinical picture.

Key words: Atrial Fibrillation. Bradycardia. Atrioventricular Block.

\section{INTRODUCCIÓN}

La génesis de la fibrilación auricular (FA) continúa siendo objeto de controversia $^{1,2}$. Probablemente corresponda a una compleja interacción entre estímulos que causan su iniciación y el sustrato anatómico auricular necesario para su perpetuación ${ }^{1}$. Se caracteriza por la presencia de intervalos R-R irregulares y ausencia de ondas $P$ definidas en el electrocardiograma (ECG) de superficie, aunque en algunas ocasiones se puede observar una cierta actividad eléctrica auricular regular en algunas derivaciones del ECG ${ }^{2}$. La longitud del ciclo auricular, cuando es visible, suele ser variable y habitualmente superior a 300 latidos por minuto $(\mathrm{lpm})^{2}$. Suele cursar con respuesta ventricular rápida. Puede ser sintomática o asintomática según la frecuencia cardiaca, la duración y la existencia o no de cardiopatía estructural ${ }^{3}$.

La frecuencia de FA se incrementa con la edad y, como consecuencia del envejecimiento progresivo de la población, la prevalencia se ha incrementado en los últimos años ${ }^{2}$. En el estudio de Framingham se observó un incremento de su prevalencia entre el periodo comprendido entre los años 1968 y 1970, y el comprendido entre 1987 y 1989, pasando de un 3,2 \% a un $9,1 \%$, respectivamente ${ }^{2}$. En los últimos años ha existido un porcentaje creciente de ingresos hospitalarios y un incremento del gasto sanitario ${ }^{2}$. La importancia de la FA se debe a que es la primera causa de eventos embólicos, especialmente de accidentes cerebrovasculares ${ }^{4}$. Dicho riesgo aumenta, según el estudio Framingham, en presencia de cardiopatía, estimando el riesgo de accidentes cerebrovasculares en un $1,5 \%$ en el grupo de edad 
comprendido entre 50 y 59 años y en un $30 \%$ en el grupo de 80-89 años ${ }^{4}$. Del mismo modo, existen unos factores de riesgo clásicos, bien definidos, implicados en el desarrollo de FA. De todos ellos, la hipertensión arterial es el principal².

\section{CASO CLÍNICO}

Varón de 79 años, que presenta obesidad, hipertensión arterial (HTA), diabetes mellitus tipo 2, cardiopatía isquémica, FA, enfermedad pulmonar obstructiva crónica (EPOC) y síndrome de apnea del sueño. Acude a consulta de Atención Primaria por presentar mareo con sensación de giro de objetos, sobre todo cuando camina, que cede en reposo. Refiere aumento de su disnea habitual. No ha presentado pérdida de consciencia, ni dolor torácico,
En la exploración física, destaca la presencia de arritmia en la auscultación cardiaca, con tonos lentos, apagados, sin soplos. Los pulsos radiales están presentes y son simétricos. En la auscultación pulmonar se aprecia hipoventilación generalizada, sin ruidos sobreañadidos. La exploración neurológica es rigurosamente normal. Presenta edemas con fóvea hasta las rodillas y signos de insuficiencia venosa crónica en miembros inferiores. Con pulsioxímetro detectamos una frecuencia cardiaca de 30 latidos por minuto (Ipm) y una saturación de oxígeno del $90 \%$.

Ante la clínica del paciente (mareo con sensación de giro de objetos, sin otra sintomatología añadida) y la frecuencia cardiaca detectada se decide realizar ECG (figura 1).

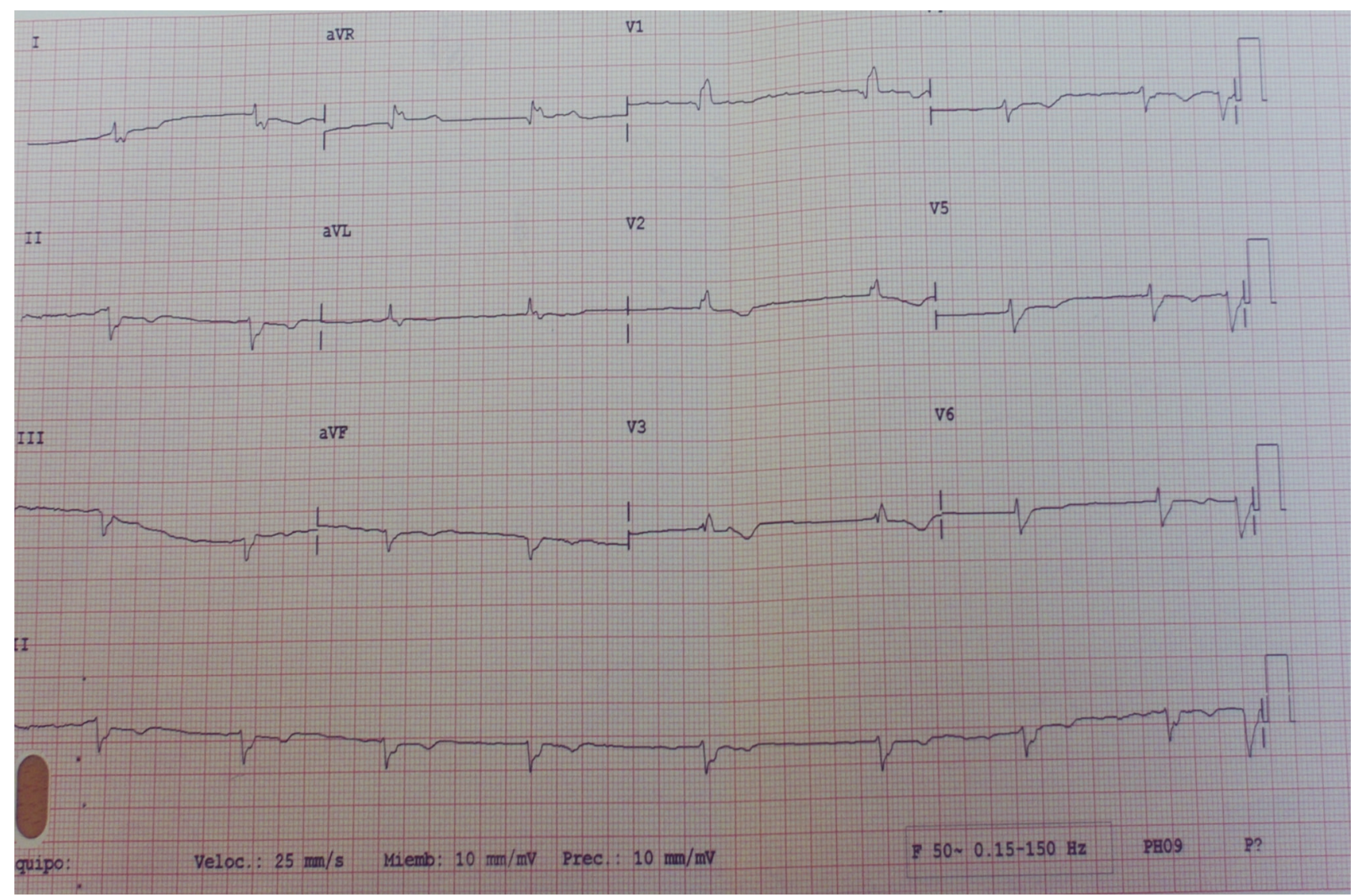

Figura 1. ECG con bradiarritmia a $40 \mathrm{lpm}$ aproximadamente, ausencia de ondas $\mathrm{P}$, eje izquierdo, bloqueo completo de rama derecha y onda T negativa V3-V6. Los complejos QRS parecen rítmicos y son anchos (escape infranodal).

ni vómitos ni sudoración fría. Realiza tratamiento habitualmente con amlodipino, repaglinida, nitroglicerina (parches), acenocumarol, atorvastatina, atenolol, ácido acetilsalicílico, espironolactona, insulina isofánica protamina, paracetamol-tramadol y omeprazol, con buen cumplimiento del mismo.
Tras valorar el ECG, y dados los antecedentes personales del paciente (HTA, cardiopatía isquémica), comparamos el registro obtenido con un ECG anterior, realizado tres años atrás durante un ingreso por un episodio de agudización de EPOC (figura 2). 


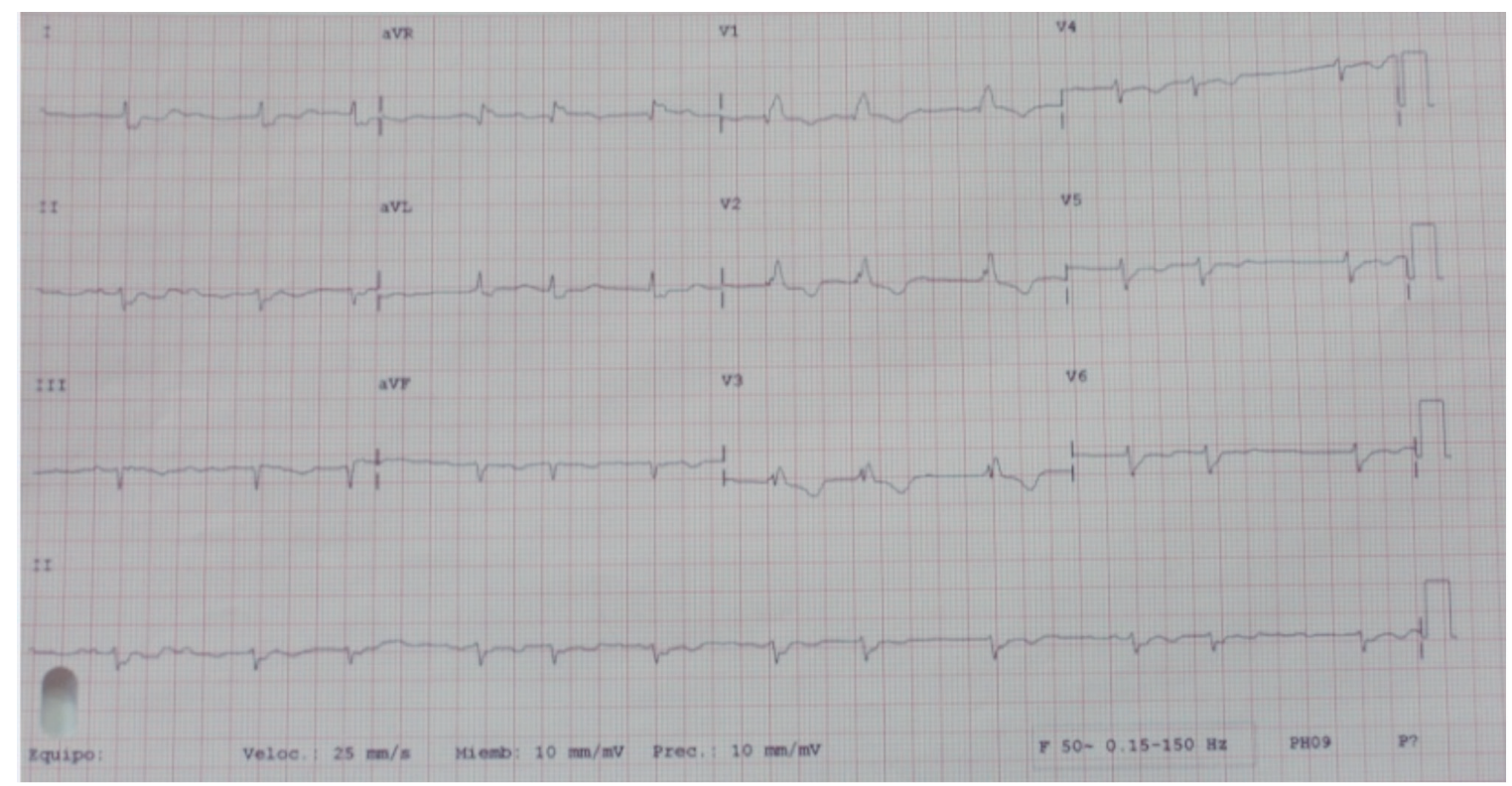

Figura 2. ECG con arritmia a $80 \mathrm{lpm}$ aproximadamente, ausencia de ondas $\mathrm{P}$, eje izquierdo, bloqueo completo de rama derecha y onda T negativa V3-V6. Los complejos QRS son arrítmicos.

Comparando ambos registros electrocardiográficos se comprueba que no aparecía bradicardia y que los complejos QRS ahora parecen rítmicos, pero no se aprecian ondas $P$, por lo que ante el ECG obtenido y la clínica se decide derivar al paciente al Servicio Hospitalario de Urgencias.

Con la sospecha diagnóstica de FA lenta, se retira el tratamiento cronotropo negativo, sin mejoría. Dada la falta de respuesta a esta medida, se decide ingreso en Cardiología. Tras estudio completo, se llega al diagnóstico definitivo de FA con bloqueo aurículo-ventricular avanzado y bloqueo de rama derecha grado III, sintomático, por lo que se decide implante de marcapasos permanente. El paciente evoluciona favorablemente: desaparece la clínica y se controla la frecuencia cardiaca.

\section{DISCUSIÓN}

La FA es la arritmia sostenida más frecuente en la práctica clínica, y es responsable de una elevada morbi-mortalidad ${ }^{1-4}$. Una FA sintomática (mareo, bradicardia) es criterio de derivación hospitalaria no demorable para realizar estudio etiológico ${ }^{4}$. Debemos pensar en la existencia de una alteración de la conducción (un bloqueo completo del nodo aurículo-ventricular que bradicardice la frecuencia apareciendo un ritmo nodal) $)^{1,4}$.

Se considera una FA lenta aquella que presenta una respuesta ventricular a menos de $60 \mathrm{lpm}$ con los complejos QRS irregulares ${ }^{1}$. A diferencia de la FA lenta, una FA con bloqueo auriculoventricular avanzado o completo muestra un silencio auricular (no se objetivan ondas $P$ en el ECG) y unos complejos QRS rítmicos que pueden ser estrechos (escape nodal) o anchos (escape infranodal) ${ }^{1}$. Las causas son las mismas que las de la FA lenta: fármacos (digoxina, betabloqueantes, calcioantagonistas), trastornos hidroelectrolíticos o hipertonía vagal'. Si se encuentra causa reversible, el tratamiento será el de dicha causa'. Si no se encuentra causa reversible, y la bradicardia es sintomática, está indicado el implante de un marcapasos definitivo ${ }^{1,2}$. El control de la frecuencia cardiaca en el paciente con FA es importante para evitar las complicaciones asociadas a la misma y controlar los síntomas².

El diagnóstico nuevo de FA lenta es, en realidad, la conocida FA del paciente con un bloqueo auriculoventricular avanzado y de rama derecha.

\section{BIBLIOGRAFÍA}

1. Rubio Alonso B, Díaz Antón B, Molina Martín de Nicolás J, Fontenla Cerezuela A. Bradiarritmias. Medicine. 2013; 11 (38) :2288-95.

2. Calvo Galiano N, García-Bolao I. Fibrilación auricular y flutter auricular. Medicine. 2013;11 (39): 2338-45.

3. Bravo Amaro M, Lago Pose E, Roca Fernández FJ. Fibrilación auricular en urgencias de Atención Primaria. Cad Aten Primaria. 2011; 18: 117-22.

4. Valdeavero Pastor M, Delgado Rubio A, Alejandre Lázaro G. Una fibrilación auricular en bradicardia. ¿Qué podemos hacer? Semergen. 2005; 31 (3): 132-5. 